\title{
S5 0836+710: An FRII jet disrupted by the growth of a helical instability?^
}

\author{
M. Perucho ${ }^{1}$, I. Martí-Vidal ${ }^{2}$, A. P. Lobanov ${ }^{3}$, and P. E. Hardee ${ }^{4}$ \\ 1 Dept. d'Astronomia i Astrofísica, Universitat de València, C/ Dr. Moliner 50, 46100 Burjassot (València), Spain \\ e-mail: manel.perucho@uv.es \\ 2 Onsala Space Observatory (Chalmers University of Technology), Observatorievägen 90, 43992 Onsala, Sweden \\ 3 Max-Planck-Institut für Radioastronomie (MPIfR), Auf dem Hügel 69, 53121 Bonn, Germany \\ ${ }^{4}$ Department of Physics \& Astronomy, The University of Alabama, Tuscaloosa, AL 35487, USA
}

Received 8 June 2012 / Accepted 25 July 2012

\begin{abstract}
Context. The remarkable stability of extragalactic jets is surprising, given the reasonable possibility of the growth of instabilities. In addition, much work in the literature has invoked this possibility to explain observed jet structures and obtain information about the jet from these structures. For example, it has been shown that the observed helical structures in the jet in S5 0836+710 could be associated with helical pressure waves generated by a Kelvin-Helmholtz instability.

Aims. Our aim is to resolve the arc-second structure of the jet in the quasar S5 0836+710 and confirm the lack of a hot-spot (reverse jet-shock) found by present observing arrays, as this lack implies a loss of jet collimation before interaction with the intergalactic medium.

Methods. We use an observation performed in 2008 using EVN (European Very Long Baseline Interferometry Network) and MERLIN (Multi-Element Radio Linked Interferometer Network). The resultant combined image after data reduction has provided a complete image of the object on arc-second scales.

Results. The lack of a hot-spot in the arc-second radio structure is taken as evidence that the jet loses its collimation between the VLBI (Very Long Baseline Interferometry) region and the region of interaction with the ambient medium.

Conclusions. This result and the previous identification of the helical structures in the jet with helical pressure waves that grow in amplitude with distance allow us to conclude that the jet is probably disrupted by the growth of Kelvin-Helmholtz instability. This observational evidence confirms that the physical parameters of jets can be extracted using the assumption that instability is present in jets and can be the reason for many observed structures. Interestingly, the observed jet is classified as a FRII (Fanaroff-Riley type II) object in terms of its luminosity, but its large-scale morphology does not correspond to this classification. The implications of this finding are discussed.
\end{abstract}

Key words. galaxies: jets - hydrodynamics - instabilities - quasars: individual: S5 0836+710

\section{Introduction}

Jets in active galactic nuclei (AGN) involve some of the most energetic processes in the Universe. The way in which they propagate and interact with the ambient medium and, in particular, their stability properties have been thoroughly studied (see Hardee 2006, 2011; Perucho 2012, for reviews). These jets are subject to the growth of different instabilities and many of the structures observed, such as knots, bendings, and helices, have been interpreted as the result of this physical process. Although there could be other ways to produce these structures, such as interactions with clumps of dense gas or the precession of the central engine, these in turn can also give rise to the growth of the instabilities via coupling to any of the unstable modes (see, e.g. Perucho et al. 2005, 2006; Mizuno et al. 2007; McKinney \& Blandford 2009; Mizuno et al. 2009; Mignone et al. 2010; Perucho et al. 2010; Mizuno et al. 2011, for numerical studies of coupling to Kelvin-Helmholtz (KH) and current-driven (CD) instabilities in relativistic flows). Our interest in understanding how this process works in jets lies in the possibility of obtaining the physical parameters of the flow (velocity, gas density, and

* FITS files are available at the CDS via anonymous ftp to cdsarc.u-strasbg.fr (130.79.128.5) or via

http://cdsarc.u-strasbg.fr/viz-bin/qcat?]/A+A/545/A65 sound speed), via modeling of the observed structures (e.g., Lobanov \& Zensus 2001; Hardee et al. 2005; Hardee \& Eilek 2011). The Very Long Baseline Interferometry (VLBI) technique has provided high-resolution observations that produce highly detailed images of the structure of AGN jets, and that even resolves them transversally (Lobanov \& Zensus 2001). Aside from those studies in which it is assumed that the observed structures are generated by instabilities, no independent proof that this is indeed the case had been provided until Perucho et al. (2012), where the authors showed that the ridge line of the jet in S5 $0836+710$ behaves as expected if it is interpreted as a pressure wave generated by KH instability. They also showed, within this framework, that the amplitude of the wave grows with distance from the core, thus fulfilling the expected characteristics of an instability.

The luminous quasar S5 $0836+710$ at a redshift $z=$ 2.16 hosts a powerful radio jet extending out to kiloparsec scales (Hummel et al. 1992). At this redshift, 1 mas $\simeq 8.4 \mathrm{pc}$ (see MOJAVE database and Wright 2006) ${ }^{1}$. VLBI monitoring of the

\footnotetext{
${ }^{1}$ https://www.physics.purdue.edu/astro/mojave/, which uses $\Lambda C D M$ Cosmology from WMAP 5 year results, and Ned Wrights Cosmology Calculator http://www.astro.ucla.edu/ wright/ CosmoCalc.html
} 
source (Otterbein et al. 1998) has yielded estimates of the bulk Lorentz factor $\gamma_{j}=12$ and the viewing angle $\alpha_{j}=3^{\circ}$ of the flow on milliarcsecond scales. The presence of an instability developing in the jet is suggested by the kink structures observed on these scales with ground VLBI (Krichbaum et al. 1990). Lobanov et al. (1998) observed the source at $5 \mathrm{GHz}$ with $\mathrm{VSOP}^{2}$ and also reported oscillations of the ridge-line. Identifying these structures with $\mathrm{KH}$ modes, they were able to derive an estimate of the basic parameters of the flow. High dynamic range VSOP and VLBA (Very Long Baseline Array of National Radio Astronomy Observatory, USA) observations of $0836+710$ at $1.6 \mathrm{GHz}$ indicated the presence of an oscillation at a wavelength as long as $\sim 100$ mas (Lobanov et al. 2006), which cannot be readily reconciled with the jet parameters given in Lobanov et al. (1998). Perucho \& Lobanov (2007) showed that the presence of a shear layer allows the fitting of all the observed oscillation wavelengths within a single set of parameters, assuming that they are produced by a KH instability growing in a cylindrical outflow. In this picture, the longest wavelength corresponds to a surface mode growing in the outer layers, whereas the shorter wavelengths are identified with body modes developing in the inner radii of the jet. In Perucho et al. (2012), the authors verified the relation between the helical structure and waves with growing amplitude.

In this paper, we present a new observation of the jet in this source using EVN+MERLIN, following the experiment presented in Hummel et al. (1992). We confirm the observation of disrupted jet structure at arc-second scales and, using our present knowledge of the source, interpret this observation.

\section{Observations}

The observations reported here were performed on 1 March 2008, and lasted 9.5 h. We used the Multi-Element Radio-Linked Interferometer Network (MERLIN, UK) ${ }^{3}$ and, simultaneously, a subset of the European VLBI Network $(\mathrm{EVN})^{4}$. On the one hand, there was a total of nine participating antennas at the EVN: Effelsberg (100 m diameter), Cambridge (32 m), Lovell (76 m), Onsala (25 m), Medicina (32 m), Noto (32 m), Torun (32 m), Urumqi (25 m), and Shanghai (25 m). On the other hand, there were seven participating antennas at MERLIN (some of them simultaneously used as EVN stations): Defford (25 m), Cambridge (32 m), Knockin (25 m), Darnhall $(25 \mathrm{~m})$, MarkII $(25 \times 38 \mathrm{~m})$, Lovell $(76 \mathrm{~m})$, and Tabley (25 m).

The observations were performed at $1.6 \mathrm{GHz}$. The recording rate per antenna at the EVN was $512 \mathrm{Mbps}$ (full polarization, 8 frequency sub-bands of $8 \mathrm{MHz}$ each, 2-bit sampling). The overall synthesized bandwidth at the EVN was $64 \mathrm{MHz}$, but the total bandwidth of MERLIN was limited to $16 \mathrm{MHz}$. The EVN data were correlated at the Joint Institute for VLBI in Europe (JIVE, the Netherlands).

We used sources 4C 39.25 and 3C345 as fringe finders, and B0859+681 as the amplitude/phase calibrator (3.9 degrees

\footnotetext{
2 VLBI Space Observatory Program, a Japanese-led space VLBI mission operated in 1997-2007 by the Institute of Space and Astronautical Science, Sagamihara, Japan http://www.vsop.isas. jaxa.jp/top.html

${ }^{3}$ MERLIN is a National Facility operated by the University of Manchester at Jodrell Bank Observatory on behalf of the Science and Technology Facilities Council.

4 The European VLBI Network is a joint facility of European, Chinese, South African, and other radio astronomy institutes funded by their national research councils.
}

of separation). The initial amplitude and phase calibration was performed with the Astronomical Image Processing system (AIPS, NRAO ${ }^{5}$ ) using standard procedures. However, the phases of the different sub-bands in the EVN observations were aligned as described in Sect. 4.1 of Martí-Vidal et al. (2012), owing to the non-constant sub-band phase offsets observed at Effelsberg and Urumqi.

Once the MERLIN and EVN observations had been independently calibrated, the data were exported to the program CASA (Common Astronomy Software Applications, NRAO), version 3.3.0, for further calibration, reduction, and imaging. We used the ms tool of CASA to scale the amplitudes of the EVN visibilities to those of MERLIN at similar baseline lengths and imaged the combined dataset with the task clean, using natural weighting of the visibilities and a multi-scale deconvolution algorithm. We used beam deconvolution scales of 7 mas (i.e., the size of the EVN synthesized beam), 170 mas (i.e., the size of the MERLIN beam), 50 mas, and 100 mas (i.e., beams of intermediate size). We also used the ms tool to apply a phase gradient (i.e., a shift in the image plane) of about 1.5 mas (south-west direction) to the EVN visibilities, to minimize the image residuals after the deconvolution (this is probably due to the effects of source structure, which may introduce small offsets in the source intensity peaks at the very different resolutions achieved with MERLIN and the EVN). Our final image (with a convolving beam of $84 \times 68$ mas; position angle of 57 degrees) is shown in Fig. 1.

The radio map presented in Fig. 1 shows an irregular structure covering a region between 1 and 2 arcsec to the southwest of the radio core. A large portion of this region falls to the west of a straight line extension of the inner jet (see Fig. 2).

\section{Discussion}

The large-scale structure is observed at distances from the radio core larger than 1 arc-second, after the jet emission disappears at 0.2 arc-seconds. This implies that there has been an increase in the particle energy at the emission site. In typical FRII jets, this happens where the jet interacts with the ambient medium, typically the intergalactic medium, and a bright reverse jet-shock (hot-spot) is observed in many of these sources. At the hot-spot, the collimated jet flow is decelerated and its particles are deflected into the radio-lobes. The bow-like shape of the radiolobes resembles the expected forward bow-shock in the ambient medium, which is difficult to observe.

In the case of S5 $0836+710$, the images of the jet on the largest scales do not show any signs of enhanced emission that could be identified as the hot-spot or any clear lobe-like structure. In contrast, the radio emission is irregular. The lack of a reverse jet-shock could imply that there has been a loss of jet collimation, as observed in FRI jets (e.g., Perucho \& Martí 2007). After the loss of collimation, the jet is transformed into a subrelativistic or mildly relativistic broad flow (Perucho et al. 2005) that continues propagating downstream and eventually interacts with the ambient medium. The observed radio emission is possibly then related to the acceleration of particles in the interaction region, which, depending on the flow velocity, could involve weak shocks.

In the case of FRI jets, the deceleration and loss of collimation has been related to mass-loading from stellar winds

\footnotetext{
5 The National Radio Astronomy Observatory is a facility of the National Science Foundation operated under cooperative agreement by Associated Universities, Inc.
} 
M. Perucho et al.: An FRII jet disrupted by the growth of a helical instability?

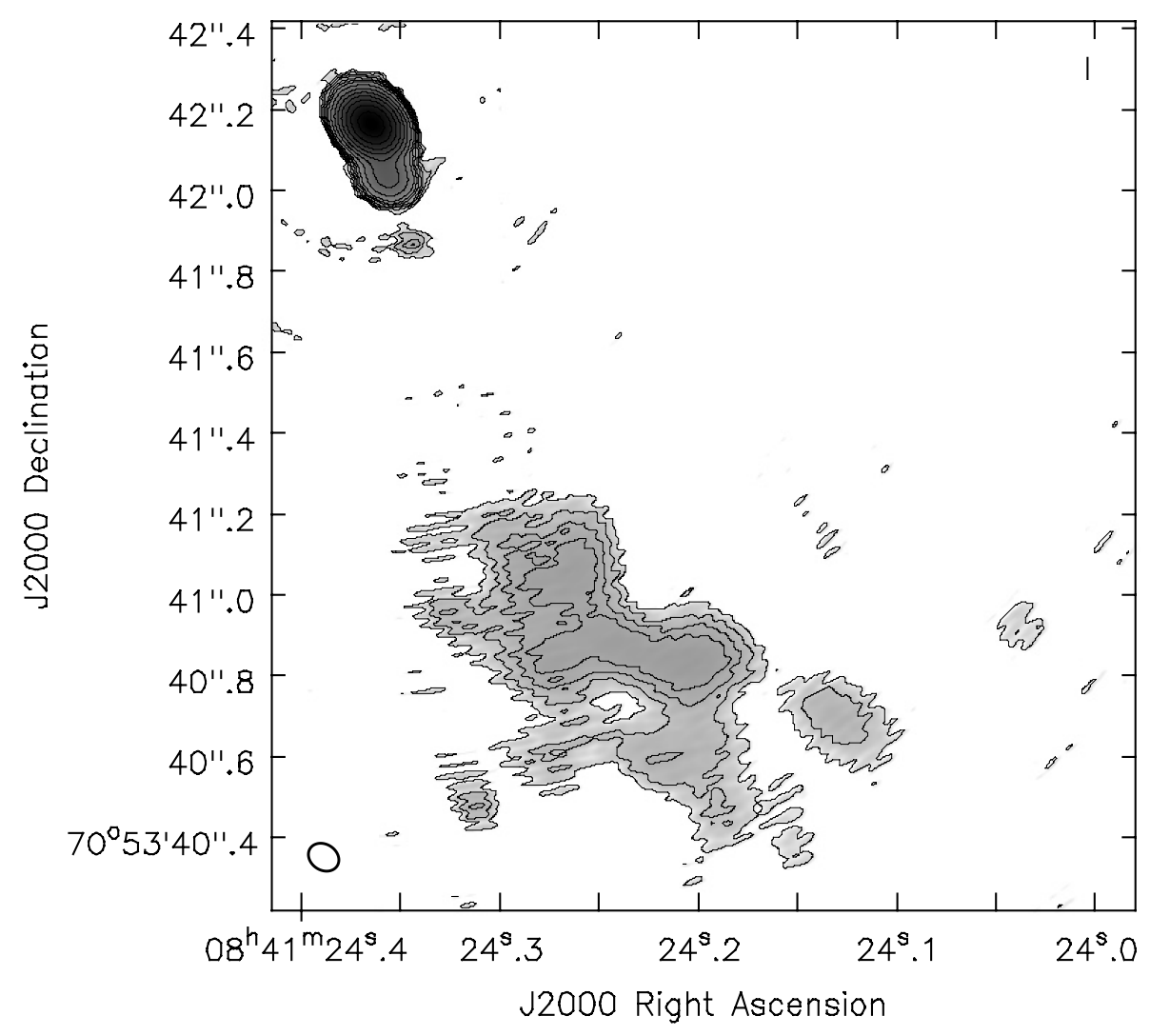

Fig. 1. EVN+MERLIN image of S5 $0836+710$ at $1.6 \mathrm{GHz}$. The contours are at $0.12,0.15,0.25$, $0.33,0.65,1.2,2.5,5,10,20,40,80$, and $99 \%$ of the image peak intensity ( $2.48 \mathrm{Jy} / \mathrm{beam})$. The convolving beam is shown at the bottom-left corner.

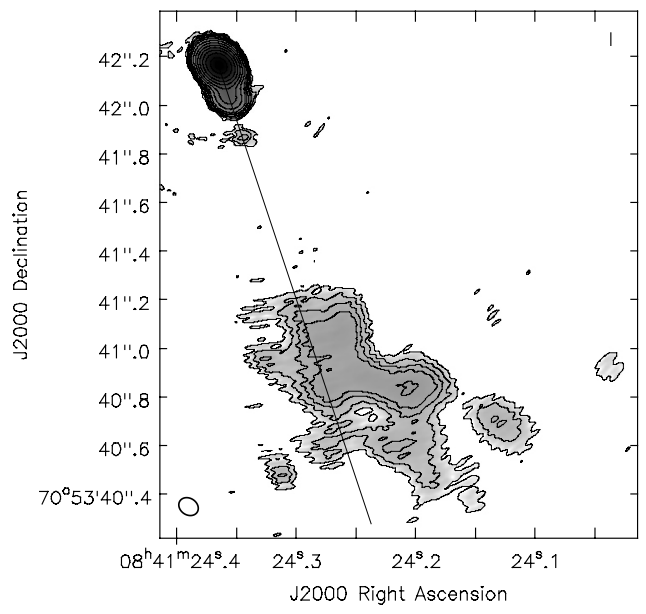

Fig. 2. Same as Fig. 1, where a straight line following the VLBI jet direction has been drawn to help the eye.

(Laing \& Bridle 2002), strong recollimation shocks (Perucho \& Martí 2007), growth of nonlinear instabilities (Rossi et al. 2008), or inhomogeneities in the ambient medium (Meliani et al. 2008). Observations seem to favor the first two possibilities in the region where FRI jets are efficiently decelerated, i.e., $\sim 1 \mathrm{kpc}$. In the case of S5 $0836+710$, the VLBI jet is observed to keep its collimation over more than 100 milli-arc-seconds, which at a viewing angle of $3^{\circ}$ and $z=2.16$ corresponds to $\sim 10 \mathrm{kpc}$. At this distance, it seems difficult that mass-loading from stellar winds, even if accumulated along the path of the jet through the host galaxy, has anything to do with the loss of collimation. In addition, there are no signs of strong shocks along the VLBI jet.

Different authors have reported kinks in the jet on different scales (see the introduction of this paper) and, in Perucho et al. (2012) the authors identify wave-like patterns in the observed helical structures. In addition, the amplitude of the helical motion increases with distance. These features are expected when an instability grows in a jet. Within this context, a small perturbation to the VLBI jet, namely a difference in pressure between two opposite sides of the jet, could couple to an unstable $\mathrm{KH}$ (if the jet is matter dominated) or $\mathrm{CD}$ (if magnetically dominated) mode that grows with distance to nonlinear amplitudes, which can lead to jet disruption and mixing with the ambient medium. There is a widely held belief that jets are dominated by particles beyond the most compact region, owing to processes such as mass-loading from stellar winds or clouds, or via the conversion of magnetic energy into kinetic energy of the particles in the process of jet acceleration (Komissarov et al. 2009). Thus, the KH scenario is favored.

A linear stability analysis using the expected parameters for the source (Perucho \& Lobanov 2007) shows that typical minimum growth lengths of disrupting, unstable modes such as the surface mode and the first body mode are $\lambda_{i} \sim 10^{2} R_{j}$. This distance provides the length after which the unstable mode amplitude is multiplied in an exponential factor. The $1.6 \mathrm{GHz}$ images in Perucho et al. (2012) show that the VLBI jet is observed out to a projected distance of $\simeq 140$ mas, which corresponds to $\simeq 22.5 \mathrm{kpc}$ at a viewing angle of $3^{\circ}$. The observed growth in amplitude from the ridge line of the $1.6 \mathrm{GHz}$ jet changes from $1-2$ mas at a projected distance of $z<50$ mas $(\simeq 8 \mathrm{kpc}$ deprojected) to 4 mas at a projected distance of $z \simeq 100-140$ mas ( $\simeq 16-22.5 \mathrm{kpc}$ deprojected). Taking into account that the amplitude of the instability evolves as $A(z)=A_{0} \exp \left(z / \lambda_{i}\right)$,

$A\left(z_{2}\right)=A\left(z_{1}\right) \frac{\exp \left(z_{2} / \lambda_{i}\right)}{\exp \left(z_{1} / \lambda_{i}\right)}$.

For $z_{1}=8 \mathrm{kpc}$, and $z_{2}=24 \mathrm{kpc}$ and using a typical jet radius of $R_{j} \simeq 20 \mathrm{mas} \simeq 170 \mathrm{pc}$ at $1.6 \mathrm{GHz}$ as obtained in Perucho \& Lobanov (2007), $A\left(z_{2}\right)=4.7$ mas if $A\left(z_{1}\right)=2$ mas. Taking into 
account that the jet and ambient properties must change over such long distances and that this has a direct influence on the growth lengths of the unstable modes, this estimate is consistent with the observations. This consistency shows that the growth of the instability obtained from the linear analysis can explain in a self-consistent way the observed growth in the amplitude of the jet ridge-line at $1.6 \mathrm{GHz}$ (Perucho et al. 2012).

This growth in the instabilities is possible because of the continuous dissipation of kinetic energy from the jet flow such that mild deceleration is expected to occur along the observed VLBI jet (see, e.g. Perucho et al. 2005, 2010; Hardee \& Eilek 2011) Deceleration and jet expansion would thus be the cause of the gap in emission between 0.2 and 1 arcsec, within our model.

The observational evidence obtained so far (Perucho et al. 2012, and here) point clearly towards this physical process taking place in the jet of S5 $0836+710$. Several helical modes, including the more disruptive low-order body modes, maybe the surface mode, and possibly some elliptical modes could be growing in the jet (Lobanov et al. 1998, 2006; Perucho et al. 2012). These wave modes generate helically twisted pressure waves, with a maximum that can be related to the peak of emission along the jet (the ridge-line, Perucho et al. 2012). Thus, we conclude that this FRII jet is probably disrupted by the growth of a helical instability. This conclusion represents the first strong observational evidence of the development of instabilities in jet flows and validates many studies that have been made under the assumption that jets develop such instabilities (e.g., Hardee 2000, 2003; Hardee et al. 2005; Hardee \& Eilek 2011).

In addition, if this FRII jet is indeed disrupted by the growth of instabilities, the immediate question is whether there are other similar cases. If there are more cases, the morphological separation between FRI and FRII jets, depending on jet power, should be reconsidered accordingly. In particular, this finding could be taken as a possible explanation for the different morphology of the jet and counter-jets in HYMORS (hybrid morphology sources, e.g., Gopal-Krishna \& Wiita 2000; Gawroński et al. 2006), if one of the two jets is perturbed and the other is not. This would require asymmetric and irregular surrounding media (under the assumption that the jet and counter-jet in a source are symmetric), but moving clouds in the broad-line and narrow-line regions can interact with the jet laterally, inducing internal shocks or sound waves that can couple to unstable modes. Observations actually show helical structures in the FRI-like side of some sources (see Gawroński et al. 2006). In the case studied here, it has been pointed out that the longest helical wavelength observed could be produced by long-term precession of the jet nozzle, with periods on the order of $T_{\mathrm{dr}} \simeq 10^{7} \mathrm{yrs}$, which is a similar value to the one given by Hardee et al. (1994) for 3C 449. Future work should be focused on trying to verify that the growing instabilities are $\mathrm{KH}$ and to obtain as much information as possible from linear perturbation theory.

Acknowledgements. M.P. acknowledges support by the Spanish "Ministerio de Ciencia e Innovación" (MICINN) grants AYA2010-21322-C03-01, AYA201021097-C03-01 and CONSOLIDER2007-00050. P.E.H. acknowledges support from NSF award AST-0908010 and NASA award NNX08AG83G to the University of Alabama.

\section{References}

Gawroński, M. P., Marecki, A., Kunert-Bajraszewska, M., \& Kus, A. J. 2006, A\&A, 447, 63

Gopal-Krishna, \& Wiita, P. J. 2000, A\&A, 363, 507

Hardee, P. E. 2000, ApJ, 533, 176

Hardee, P. E. 2003, ApJ, 597, 798

Hardee, P. E. 2006, in Relativistic Jets: The Common Physics of AGN, Microquasars and Gamma-Ray Bursts, eds. P. A. Hughes, \& J. N. Bregman, AIP Conf. Proc., 856, 57

Hardee, P. E. 2011, in Jets at all Scales, eds. G. Romero, R. Sunyaev, \& T. Belloni, IAU Symp., 275, 41

Hardee, P. E., \& Eilek, J. A. 2011, ApJ, 735, 61

Hardee, P. E., Cooper, M. A., \& Clarke, D. A. 1994, ApJ, 424, 126

Hardee, P. E., Walker, R. C., \& Gómez, J. L. 2005, ApJ, 620, 646

Hummel, C. A., Mruxlow, T. W. B., Krichbaum, T. P., et al. 1992, A\&A, 266, 93

Komissarov, S. S., Vlahakis, N., Königl, A., \& Barkov, M. V. 2009, MNRAS, 394, 1182

Krichbaum, T. P., Hummel, C. A., Quirrenbach, A., et al. 1990, A\&A, 230, 271

Laing, R. A., \& Bridle, A. H. 2002, MNRAS, 336, 328

Lobanov, A. P., \& Zensus, J. A. 2001, Science, 294, 128

Lobanov, A. P., Krichbaum, T. P., Witzel, A., et al. 1998, A\&A, 340, 60

Lobanov, A. P., Krichbaum, T. P., Witzel, A., \& Zensus, J. A. 2006, PASJ, 58, 253

Martí-Vidal, I., Krichbaum, T. P., Marscher, A., et al. 2012, A\&A, 542, A107

McKinney, J. C., \& Blandford, R. D. 2009, MNRAS, 394, L126

Meliani, Z., Keppens, R., \& Giacomazzo, B. 2008, A\&A, 491, 321

Mignone, A., Rossi, P., Bodo, G., Ferrari, A., \& Massaglia, S. 2010, MNRAS, 402, 7

Mizuno, Y., Hardee, P. E., \& Nishikawa, K.-I. 2007, ApJ, 662, 835

Mizuno, Y., Lyubarsky, Y., Nishikawa, K.-I., \& Hardee, P. E. 2009, ApJ, 700, 684

Mizuno, Y., Hardee, P. E., \& Nishikawa, K.-I. 2011, ApJ, 734, 19

Otterbein, K., Krichbaum, T. P., Kraus, A., et al. 1998, A\&A, 334, 489

Perucho, M. 2012, Int. J. Mod. Phys. Conf. Ser., 8, 241

Perucho, M., \& Lobanov, A. P. 2007, A\&A, 469, L23; Erratum, 2011, 533, C2

Perucho, M., \& Martí, J. M. 2007, MNRAS, 382, 526

Perucho, M., Martí, J. M., \& Hanasz, M. 2005,A\&A, 443, 863

Perucho, M., Lobanov, A. P., Martí, J. M., \& Hardee, P. E. 2006, A\&A, 456, 493

Perucho, M., Martí, J. M., Cela, J. M., et al. 2010, A\&A, 519, A41

Perucho, M., Kovalev, Y. Y., Lobanov, A. P., Hardee, P. E., \& Agudo, I. 2012, ApJ, 749, 55

Rossi, P., Mignone, A., Bodo, G., Massaglia, S., \& Ferrari, A. 2008, A\&A, 488, 795

Wright, N. 2006, PASP, 118, 1711 